\title{
Nuclear Stellar Disks in E/S0 Galaxies
}

\author{
Walter Jaffe, Frank van den Bosch \\ Leiden Observatory, P.O. 9513, Leiden, 2300RA, Netherlands
}

\begin{abstract}
New HST images and spectra of the nuclear disks in two Virgo Cluster E/S0 galaxies reveal that they were probably formed together with their parent galaxies. The two galaxies show very different color gradients. This is most likely caused by the escape of high metallicity gas from early stars in the lower mass galaxy. One galaxy shows strong kinematic evidence for a massive central black hole.
\end{abstract}

\section{Background}

In an earlier HST survey of the nuclear structures of early type galaxies in the Virgo cluster we discovered a novel morphological structure: four out of the fourteen galaxies observed showed bright, thin stellar disks in their nuclei (Jaffe et al. 1994, Ferrarese et al. 1994, v.d. Bosch et al. 1994). The disks are very small; their radial exponential scale length is typically $15 \mathrm{pc}$ and their thickness less than $10 \mathrm{pc}$. They seem only to appear in relatively faint galaxies with $\mathrm{M}_{B}<-19$. There were eight faint galaxies in the survey, so the rate of occurance of disks in the fainter galaxies is at least of order $50 \%$ and may approach $100 \%$ since disks seen face-on might not be recognized. The fainter host galaxies, which we designated as "Type II" often have S0 type outer disks at kpc scales, are usually in rapid rotation, and have extremely bright central regions: the central visual surface brightness is as high as $13.5 \mathrm{mag} / \mathrm{arcsec}^{2}$ in contrast to the larger pressure supported elliptical galaxies where the central brightness seldom exceeds $16.5 \mathrm{mag} / \mathrm{arcsec}^{2}$.

\section{The Current Program}

The disks should serve as indicators of the history of these fainter galaxies in clusters. If they have been formed by infall of gaseous material into predominately stellar galaxies, this should leave its mark in the color and metallicity of the disks, and we can determine the importance of this phenomena in cluster evolution. If the disks were formed early on, the persistence of such a dynamically cold subsystem limits the rate of collisions and near collisions between cluster galaxies. We can also ask what dynamical effects have led to the existence of two distinct disk systems: the inner nuclear disk and the outer $\mathrm{S} 0$ disk.

They may also provide clues to the evolution of the more massive "TypeI" galaxies. It is at least plausible that these have been formed by mergers of 
Type-II precursors. Specifying the dynamic properties of the Type-II galaxies will allow detailed modeling of the mergers so see if the remnants resemble the fat Type-I galaxies in dynamics and populations.

In addition to these evolutionary questions, the disks are a useful probe of the mass distribution of the nuclei of these fainter galaxies. Since the disks are bright and in simple rotation, it is (relatively) straightforward to go from measured Doppler velocities to estimates of central masses.

\section{Observations}

To investigate these issues we proposed and received new HST observations of two of the disk galaxies: NGC 4570 and NGC 4342 .

- Planetary Camera-2 images in U, V, and I bands (after correction of HST spherical aberration). These allow isophotal analysis of the disk structures at higher resolution and reliability than the old images, and construction of color gradient maps.

- Faint Object Spectrometer spectra at 0.25 " resolution to measure $\mathrm{Mg}$ and $\mathrm{H} \beta$ metallicity indicators, and to measure the rotation and dispersion velocities along the major and minor axes.

In addition William Herschel Telescope (WHT) spectra at 0.8 " resolution were taken to tie the HST kinematic information to the dynamics at larger radii.

\section{Preliminary Results}

This is very much work in progress and while we have all of our HST data, we report here only very preliminary results.

The new PC-2 images confirm that all the structures we identified on the original PC-1 images are real and not artifacts of the image enhancement techniques used to (partially) correct for the spherical aberrations. While the overall appearance of the two galaxies are quite similar, the color-color $(U-V$ and $V-I)$ maps of the two galaxies are remarkably different. NGC 4342 shows essentially no color gradients, while NGC 4570 shows a gradient of $1 \mathrm{mag}$ in $U-V$ and 0.2 mag in $V-I$. The latter galaxy is much redder in its central regions. In both cases the disk has exactly the same colors as the bulge stars at the same radius.

The large gradient in NGC 4570 can be most easily explained by its higher mass. If the redder nuclear regions are caused by higher metallicity this could be caused by trapping there of metals from early stellar generations, which escaped from the lower potential field of NGC 4342. Apparently the disks are formed coevally with the host galaxies, and not by later capture. We tried to determine by comparison with theoretical isochrones whether the color gradients where caused by age or metallicity gradients, but it is well known that these are difficult to distinguish and we found that different authors' isochrones yielded entirely different interpretations of our colors.

Analysis of the rotation and dispersion curves of NGC 4342 indicates high velocities and very high central velocity dispersions. The dispersion rises to 320 
$\mathrm{km} \mathrm{s}^{-1}$ at the innermost WHT point, and $418 \mathrm{~km} \mathrm{~s}^{-1}$ at the innermost HST point (corresponding to $20 \mathrm{pc}$ ). These are very hard to explain without invoking a central black hole of several $\times 10^{8} M_{\odot}$. This conclusion is not yet definitive because the light from this region is diluted by emission from a large number of bulge stars. Correction for their contribution to the dispersion involves careful modeling of their orbital distributions, which is currently being undertaken (v.d. Bosch and de Zeeuw, 1996). It is surprising that black holes of this large mass seem to be present in low mass, totally quiescent galaxies.

A final interesting point is that the nuclear stellar disks, if plotted on a surface brightness-scale length plot, form a perfect continuation of the locus of points formed by (from largest to smallest): giant spiral galaxy disks, S0 disks, and disk-like structures in E/S0 galaxies. This seems to point to a universal mechanism limiting their brightness- perhaps a kinematic instability.

\section{Conclusions}

- Nuclear disks are common in fainter E/S0 galaxies.

- Disks are cold rotational systems. Disky galaxies are happy hunting grounds for black holes.

- Nuclear disks form a continuum with disky elliptical/S0/Sp galaxies in a brightness/diameter plot.

- Outer Disks/Bulges/Nuclear Disks show a smooth transition of colors, and were probably formed almost coevally.

- Many (most? all?) intermediate brightness E/S0 galaxies probably have massive nuclear black holes.

\section{Questions}

- Why aren't Type-II galaxies active?

- Why is there a inner/outer disk morphology?

- Why are the inner disks so cold?

- What does the surface brightness/diameter plot mean?

- Can you form fat ellipticals from mergers of skinny galaxies?

- Can you ever say anything definitive from color-color diagrams or from stellar dynamics?

\section{References}

Ferrarese, L, v.d. Bosch, F.C., Jaffe, W., Ford, H.C., O'Connell, R.W. 1994, AJ, 108, 1598 
Jaffe, W., Ford, H.C., O'Connell, R.W, v.d.Bosch, F.C., Ferrarese, L, 1994, AJ, 108,1567

v.d. Bosch, F.C., Ferrarese, L, Jaffe, W., Ford, H.C., O'Connell, R.W. 1994, AJ, 108, 1579

v.d. Bosch, F.C., de Zeeuw, P.T., MNRASin press 